\title{
Analytical study of nonlinear oscillatory systems using the Hamiltonian approach technique
}

\author{
Martin Hermann • Masoud Saravi • \\ Hadi Ebrahimi Khah
}

Received: 22 September 2013/ Accepted: 20 May 2014/Published online: 6 June 2014

(c) The Author(s) 2014. This article is published with open access at Springerlink.com

\begin{abstract}
In this article, we investigate and apply Hamiltonian approach method as one of the analytical approximate techniques, for studying the strongly nonlinear dynamical systems such as the motion of a rigid rod rocking back on the circular surface without slipping and the free vibrations of an autonomous conservative oscillator with inertia and static-type fifth-order nonlinearities. To illustrate the applicability and accuracy of the method, the approximate solution results are compared with exact and numerical solutions.
\end{abstract}

Keywords Dynamical systems - Strongly nonlinear differential equations - Analytical approximate techniques . Hamiltonian approach method · Numerical method . Runge-Kutta method

\section{Introduction}

Most of the dynamical systems facing engineers, physicists and applied mathematicians today exhibit certain essential features which preclude exact analytical solutions. Some of these features are nonlinearities, variable coefficients, complex boundary shapes, and nonlinear boundary conditions at

M. Hermann · H. Ebrahimi Khah ( $\varangle)$

Fakultät für Mathematik und Informatik, Friedrich-Schiller-

Universität Jena, 07743 Jena, Germany

e-mail: hadi_ebrahimi2002@yahoo.com

M. Hermann

e-mail: prof@martin-hermann.de

M. Saravi

Department of Mathematics, Islamic Azad University Nour

Branch, 731 Nour, Iran

e-mail: masoud@saravi.info known or, in some cases, unknown boundaries. Even if the exact solution of a problem can be found explicitly, it may be useless for mathematical and physical interpretation or numerical evaluation. Examples of such problems are Bessel functions of large argument and large-order and doubly periodic functions. Therefore, to obtain information about solutions of equations, we are forced to resort to approximate and numerical solutions, or combinations of both.

Recently, considerable attention has been directed toward the analytical approximate solutions for the strongly nonlinear differential equations of dynamical systems. The traditional perturbation methods have many shortcomings, and they are not valid for strongly nonlinear dynamical systems.

To overcome the shortcomings, many techniques have appeared in open literature, for example, parameterexpanding method [1-3], variational iteration method [47], energy balance method [8-14], variational approach method [15-19], amplitude-frequency formulation [2022], homotopy perturbation method [23-29], and the other analytical approximate solutions [30].

The solution of differential equations in physics and engineering, especially some oscillation equations are nonlinear, and in most cases it is difficult to solve such equations, especially analytically. Previously, He had introduced the energy balance method based on collocation and the Hamiltonian. This method can be seen as a Ritz method and leads to a very rapid convergence of the solution, and can be easily extended to other nonlinear oscillations.

This approach is very simple but strongly depends upon the chosen location point. Recently, He [31] has proposed the Hamiltonian approach to overcome the shortcomings of the energy balance method. This approach is a kind of energy method with a vast application in conservative oscillatory systems. Application of this method can be found in many literatures [31-35]. 
The Hamiltonian approach method is the subject of this article, as one of the analytical approximate techniques. In this article, we present two examples to illustrate the applicability, accuracy and effectiveness of the Hamiltonian approach method as one of the analytical approximate techniques.

As the first example in this paper, we investigate the nonlinear differential equation of the motion of a rigid rod rocking back on the circular surface without slipping [36]. In the second example, we investigate the nonlinear differential equation of the free vibrations of an autonomous conservative oscillator with inertia and static-type fifthorder nonlinearities [37].

To illustrate the accuracy of the Hamiltonian approach method, in first example, we compare the approximate solution result with exact solution. In second example, because there is no exact solution, we compare the approximate solution result with Runge-Kutta method as one of the known numerical methods.

\section{The description of Hamiltonian approach method}

To descript the He's Hamiltonian approach method, we consider the following general oscillator [31]:

$\frac{\mathrm{d}^{2}}{\mathrm{~d} t^{2}} u(t)+f(u(t))=0$

with initial conditions:

$u(0)=A, \frac{\mathrm{d}}{\mathrm{d} t} u(0)=0$.

It is easy to establish a variational principle for Eq. (1), which reads [31]:

$J(u(t))=\int_{0}^{T / 4}\left\{-\frac{1}{2}\left(\frac{\mathrm{d}}{\mathrm{d} t} u(t)\right)^{2}+F(u(t))\right\} \mathrm{d} t$

where $T$ is the period of the oscillator $\partial F / \partial u=f(u)$.

In the functional (3), $-\frac{1}{2}\left(\frac{\mathrm{d}}{\mathrm{d} t} u(t)\right)^{2}$ is kinetic energy, and $F(u(t))$ is potential energy, so the functional (3) is the least Lagrangian action, from which we can immediately obtain its Hamiltonian, which reads:

$H=\frac{1}{2}\left(\frac{\mathrm{d}}{\mathrm{d} t} u(t)\right)^{2}+F(u(t))=\mathrm{constan} \mathrm{t}=H_{0}$

or:

$R(t)=\frac{1}{2}\left(\frac{\mathrm{d}}{\mathrm{d} t} u(t)\right)^{2}+F(u(t))-F(A)$

Equation (4) implies that the total energy keeps unchanged during the oscillation.
We use the following trial function to determine the angular frequency $\omega$.

$u(t)=A \cos \omega t$

where $\omega$ is the frequency. Submitting Eq. (6) into Eq. (5) results in a residual:

$R(t)=\frac{1}{2} A^{2} \omega^{2} \sin ^{2} \omega t+F(A \cos \omega t)-F(A)$

If, by chance, the exact solution had been chosen as the trial function, then it would be possible to make $R$ zero for all values of $t$ by appropriate choice of $\omega$. Since $u(t)=A \cos \omega t$ is an approximation to the exact solution, $R$ cannot be made zero everywhere. According to the energy balance method [2], locating at some a special point, that is, $\omega t=\pi / 4$ and setting $R(t=\pi / 4 \omega)=0$, we can obtain an approximate frequencyamplitude relationship of the studied nonlinear oscillator. Such treatment is much simple and has been widely used by engineers [3-7]. The accuracy of such location method, however, strongly depends upon the chosen location point. To overcome the shortcoming of the energy balance method, in this paper, we apply a new approach based on Hamiltonian $[8,9]$.

From Eq. (7), we have:

$\frac{\partial H}{\partial A}=0$.

Introducing a new function, $\bar{H}(u(t))$, defined as:

$\bar{H}(u(t))=\int_{0}^{T / 4}\left\{\frac{1}{2}\left(\frac{\mathrm{d}}{\mathrm{d} t} u(t)\right)^{2}+F(u(t))\right\} \mathrm{d} t=\frac{1}{4} T H$

It is obvious that:

$\frac{\partial \bar{H}}{\partial T}=\frac{1}{4} H$

Equation (10) is, then, equivalent to the following one:

$\frac{\partial}{\partial A}\left(\frac{\partial \bar{H}}{\partial T}\right)=0$

or

$\frac{\partial}{\partial A}\left(\frac{\partial \bar{H}}{\partial(1 / \omega)}\right)=0$.

From Eq. (12), we can obtain approximate frequencyamplitude relationship of a nonlinear oscillators.

\section{The application of Hamiltonian approach method for nonlinear dynamical systems}

The motion of a rigid rod rocking back on the circular surface without slipping

In this section, we consider the motion of a rigid rod rocking back on the circular surface without slipping. The 
governing equation of this motion is in the following form [36]:

$$
\begin{aligned}
& \left(\frac{\mathrm{d}^{2}}{\mathrm{~d} t^{2}} u(t)\right)+\frac{3}{4} u(t)^{2}\left(\frac{\mathrm{d}^{2}}{\mathrm{~d} t^{2}} u(t)\right) \\
& \quad+\frac{3}{4} u(t)\left(\frac{\mathrm{d}}{\mathrm{d} t} u(t)\right)^{2}+3 \frac{g}{l} u(t) \cos u(t)=0
\end{aligned}
$$

with the following initial conditions [36]:

$$
u(0)=A, \frac{\mathrm{d}}{\mathrm{d} t} u(0)=0
$$

where $g$ and $l$ are the positive constants. For this problem, we have:

$$
\begin{aligned}
f(u(t))= & \frac{3}{4} u(t)^{2}\left(\frac{\mathrm{d}^{2}}{\mathrm{~d} t^{2}} u(t)\right) \\
& +\frac{3}{4} u(t)\left(\frac{\mathrm{d}}{\mathrm{d} t} u(t)\right)^{2}+3 \frac{g}{l} u(t) \cos u(t)
\end{aligned}
$$

and:

$F(u(t))=\frac{3}{8} u(t)^{2}\left(\frac{\mathrm{d}}{\mathrm{d} t} u(t)\right)^{2}+\frac{3 g(\cos u(t)+u(t) \sin u(t))}{l}$.

Its Hamiltonian can be easily obtained, which reads:

$$
\begin{aligned}
H= & \frac{1}{2}\left(\frac{\mathrm{d}}{\mathrm{d} t} u(t)\right)^{2}+\frac{3}{8} u(t)^{2}\left(\frac{\mathrm{d}}{\mathrm{d} t} u(t)\right)^{2} \\
& +\frac{3 g(\cos u(t)+u(t) \sin u(t))}{l} .
\end{aligned}
$$

Integrating Eq. (17) with respect to $t$ from 0 to $T / 4$, we have:

$$
\begin{aligned}
\bar{H}(u(t))= & \int_{0}^{T / 4}\left\{\frac{1}{2}\left(\frac{\mathrm{d}}{\mathrm{d} t} u(t)\right)^{2}+\frac{3}{8} u(t)^{2}\left(\frac{\mathrm{d}}{\mathrm{d} t} u(t)\right)^{2}\right. \\
& \left.+\frac{3 g(\cos u(t)+u(t) \sin u(t))}{l}\right\} \mathrm{d} t .
\end{aligned}
$$

Assuming that the solution can be expressed as $u(t)=A \cos \omega t$ and substituting it to Eq. (18), we obtain:

$$
\begin{aligned}
\bar{H}(u(t)) & =\int_{0}^{T / 4}\left\{\begin{array}{l}
\frac{1}{2} A^{2} \omega^{2} \sin ^{2} \omega t+\frac{3}{8} A^{4} \omega^{2} \cos ^{2} \omega t \sin ^{2} \omega t \\
+\frac{3 g(\cos (A \cos \omega t)+A \cos \omega t \cdot \sin (A \cos \omega t))}{l}
\end{array}\right\} \mathrm{d} t \\
& =\int_{0}^{\pi / 2}\left\{\begin{array}{l}
\frac{1}{2} A^{2} \omega \sin ^{2} t+\frac{1}{\omega}\left[\begin{array}{l}
\frac{3}{8} A^{4} \omega^{2} \cos ^{2} t \sin ^{2} t \\
\left.+\frac{3 g(\cos (A \cos t)+A \cos t \cdot \sin (A \cos t))}{l}\right]
\end{array}\right] \\
\end{array}=\frac{1}{2} A^{2} \omega \cdot \frac{\pi}{4}+\frac{1}{\omega}\left[\frac{3}{8} A^{4} \omega^{2} \cdot \frac{\pi}{16}+\frac{\pi}{2} \cdot \frac{3 g(\operatorname{Bessel} \mathrm{J}(0, \mathrm{~A})+A \cdot \operatorname{Bessel} \mathrm{J}(1, \mathrm{~A}))}{l}\right] .\right.
\end{aligned}
$$

In Eq. (19), we have two Bessel functions of the first kind. The Bessel functions are one of the special functions in mathematics. We can expand these Bessel functions of the first kind in the following form:

$\operatorname{Bessel} \mathrm{J}(0, A)=1-\frac{1}{4} A^{2}+\frac{1}{64} A^{4}$

and:

Bessel $\mathrm{J}(1, A)=\frac{1}{2} A-\frac{1}{16} A^{3}+\frac{1}{384} A^{5}$

By substituting Eqs. (20) and (21) into Eq. (19), we obtain:

$$
\begin{aligned}
\bar{H}(u(t))= & \frac{1}{2} A^{2} \omega \cdot \frac{\pi}{4}+\frac{1}{\omega} \\
& \times\left\{\frac{3}{8} A^{4} \omega^{2} \cdot \frac{\pi}{16}+\frac{\pi}{2} \cdot \frac{3 g}{l}\left[\begin{array}{l}
\left(1-\frac{1}{4} A^{2}+\frac{1}{64} A^{4}\right) \\
+A \cdot\left(\frac{1}{2} A-\frac{1}{16} A^{3}+\frac{1}{384} A^{5}\right)
\end{array}\right]\right\}
\end{aligned}
$$

Setting:

$$
\begin{aligned}
\frac{\partial}{\partial A}\left(\frac{\partial \bar{H}}{\partial(1 / \omega)}\right)= & -A \omega^{2} \cdot \frac{\pi}{4}-\frac{3}{8} A^{3} \omega^{2} \cdot \frac{\pi}{4}+\frac{3 g}{l} \\
& \cdot \frac{\pi}{2}\left(-\frac{1}{2} A+\frac{1}{16} A^{3}+A-\frac{1}{4} A^{3}+\frac{1}{64} A^{5}\right) \\
= & 0
\end{aligned}
$$

We obtain the following frequency-amplitude relationship:

$\omega_{\text {HAM }}=\frac{\sqrt{(3 g / l)\left(32+12 A^{2}\right)\left(32-12 A^{2}+A^{4}\right)}}{32+12 A^{2}}$.

Its period can be written in the following form:

$$
T_{\text {HAM }}=\frac{2 \pi}{\frac{\sqrt{(3 g / l)\left(32+12 A^{2}\right)\left(32-12 A^{2}+A^{4}\right)}}{32+12 A^{2}}} .
$$

To illustrate the accuracy of the Hamiltonian approach method, we compare the approximate solution results with exact solution. For this dynamical system, the exact period is in the following form [36]:

$$
\begin{aligned}
T_{\text {Exact }}= & 4\left(\frac{l}{3 g}\right)^{1 / 2} \int_{0}^{\pi / 2} \\
& \times\left(\frac{\left(4+3 A^{2} \sin ^{2} \varphi\right) A^{2} \cos ^{2} \varphi}{8[A \sin A+\cos A-A \sin \varphi \sin (A \sin \varphi)-\cos (A \sin \varphi)]}\right)^{1 / 2} \mathrm{~d} \varphi .
\end{aligned}
$$

A comparison of obtained results from the approximate period and the exact one is tabulated in Table 1 for $g=1.00, l=1.00$ and different values of $A$. From Table 1, the maximum relative error of the approximate periods is $1.9344 \%$ for $g=1.00, l=1.00$ and $A=0.45 \pi$.

Also, we present the comparison results of analytical approximate solution of $u(t)$ based on $t$ with exact solution 
Table 1 Comparison of approximate periods with exact periods for $g=1.00, l=1.00$ and different values of $A$

\begin{tabular}{llll}
\hline$A$ & $\begin{array}{l}\text { Hamiltonian } \\
\text { approach method }\end{array}$ & $\begin{array}{l}\text { Exact } \\
\text { solution }\end{array}$ & $\begin{array}{l}\text { Error } \\
\text { percentage }\end{array}$ \\
\hline $0.05 \pi$ & 3.66126 & 3.66109 & 0.0054 \\
$0.10 \pi$ & 3.76398 & 3.76397 & 0.0008 \\
$0.15 \pi$ & 3.94065 & 3.94086 & 0.0056 \\
$0.20 \pi$ & 4.20182 & 4.20292 & 0.0264 \\
$0.25 \pi$ & 4.56433 & 4.56948 & 0.1129 \\
$0.30 \pi$ & 5.05832 & 5.07728 & 0.3734 \\
$0.35 \pi$ & 5.76741 & 5.79770 & 0.5224 \\
$0.40 \pi$ & 6.73586 & 6.81564 & 1.1705 \\
$0.45 \pi$ & 8.67226 & 8.84333 & 1.9344 \\
\hline
\end{tabular}

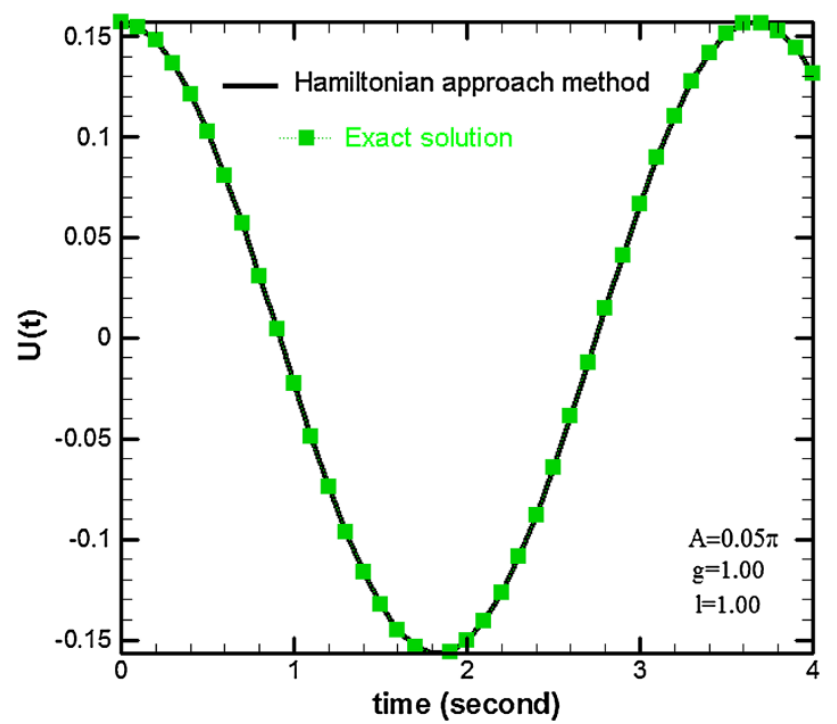

Fig. 1 Comparison of Hamiltonian approach method solution of $u(t)$ based on $t$ with exact solution for $g=1.00, l=1.00, A=0.05 \pi$

with $g=1.00, l=1.00$ and different values of $A$ in Figs. 1 and 2 .

Also, to investigate on the behavior of this dynamical system, the effect of parameters $g$ and $l$ on the frequency corresponding to different values of amplitude $(A)$ has been studied in Figs. 3 and 4.

The free vibrations of an autonomous conservative oscillator with inertia and static-type fifth-order nonlinearities

In this section, we consider the free vibrations of an autonomous conservative oscillator with inertia and statictype fifth-order nonlinearities. The differential equation of this dynamical system is the following form [37]:

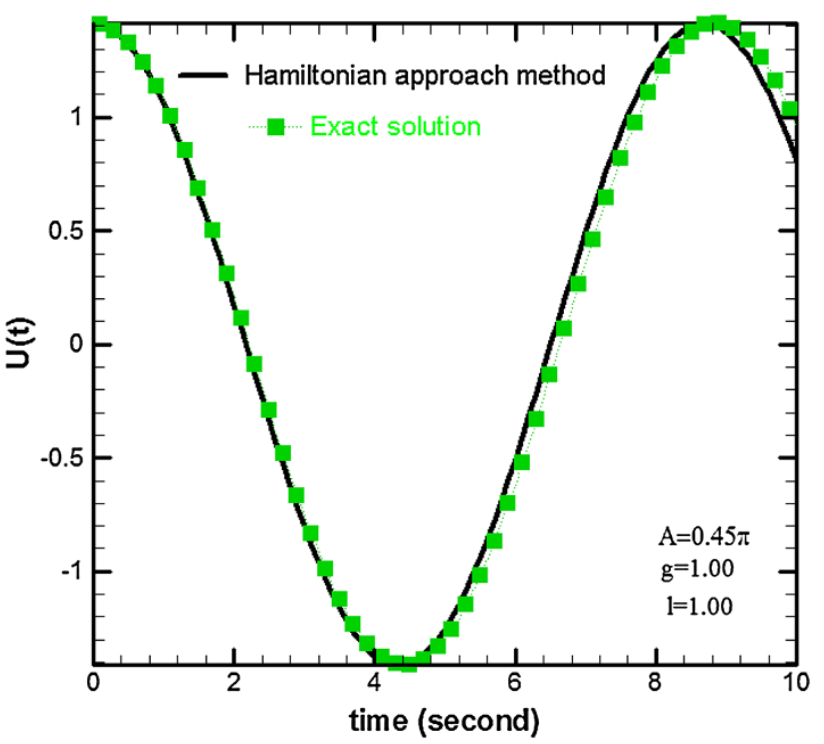

Fig. 2 Comparison of Hamiltonian approach method solution of $u(t)$ based on $t$ with exact solution for $g=1.00, l=1.00, A=0.45 \pi$

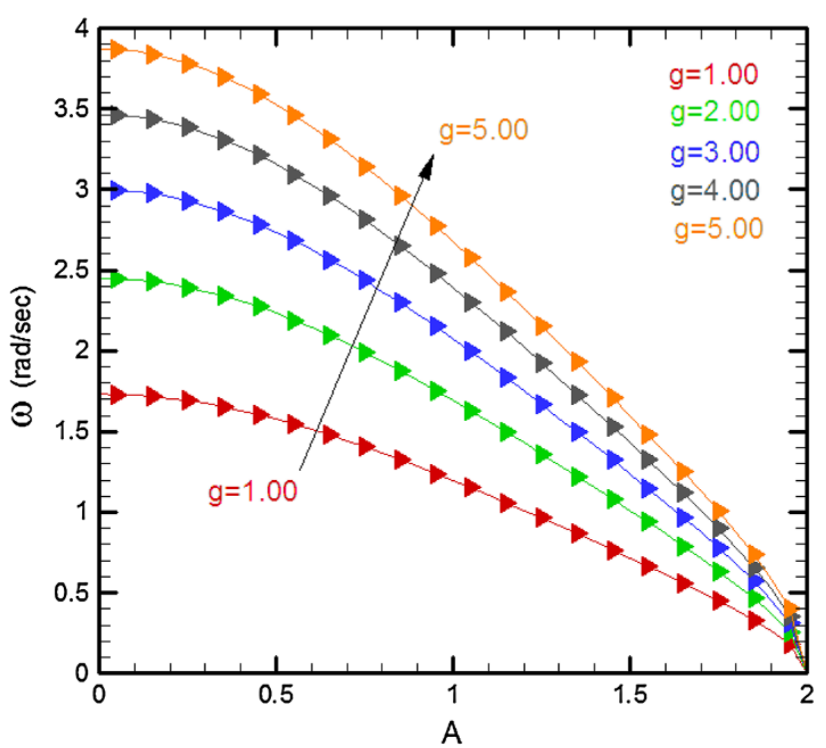

Fig. 3 Comparison of frequency corresponding to different values of amplitude $(A)$ and $l=1.00$

$$
\begin{gathered}
\left(\frac{\mathrm{d}^{2}}{\mathrm{~d} t^{2}} u(t)\right)+\varepsilon_{1} u(t)^{2}\left(\frac{\mathrm{d}^{2}}{\mathrm{~d} t^{2}} u(t)\right)+\varepsilon_{2} u(t)^{4}\left(\frac{\mathrm{d}^{2}}{\mathrm{~d} t^{2}} u(t)\right)+\varepsilon_{1} u(t)\left(\frac{\mathrm{d}}{\mathrm{d} t} u(t)\right)^{2} \\
\quad+2 \varepsilon_{2} u(t)^{3}\left(\frac{\mathrm{d}}{\mathrm{d} t} u(t)\right)^{2}+\lambda u(t)+\varepsilon_{3} u(t)^{3}+\varepsilon_{4} u(t)^{5}=0, \quad-1 \leq \lambda \leq 1
\end{gathered}
$$

with the following initial conditions [37]:

$$
u(0)=A, \frac{\mathrm{d}}{\mathrm{d} t} u(0)=0
$$

where $\varepsilon_{1}, \varepsilon_{2}, \varepsilon_{3}$ and $\varepsilon_{4}$ are positive parameters and $\lambda$ is an integer which may take values of $-1,0$ and 1 [37]. For this problem, we have: 


$$
\begin{aligned}
f(u(t))= & \varepsilon_{1} u(t)^{2}\left(\frac{\mathrm{d}^{2}}{\mathrm{~d} t^{2}} u(t)\right)+\varepsilon_{2} u(t)^{4}\left(\frac{\mathrm{d}^{2}}{\mathrm{~d} t^{2}} u(t)\right)+\varepsilon_{1} u(t)\left(\frac{\mathrm{d}}{\mathrm{d} t} u(t)\right)^{2} \\
& +2 \varepsilon_{2} u(t)^{3}\left(\frac{\mathrm{d}}{\mathrm{d} t} u(t)\right)^{2}+\lambda u(t)+\varepsilon_{3} u(t)^{3}+\varepsilon_{4} u(t)^{5}
\end{aligned}
$$

and:

$$
\begin{aligned}
F(u(t))= & \frac{1}{2}\left(\frac{\mathrm{d}}{\mathrm{d} t} u(t)\right)^{2} \varepsilon_{1} u(t)^{2}+\frac{1}{2}\left(\frac{\mathrm{d}}{\mathrm{d} t} u(t)\right)^{2} \varepsilon_{2} u(t)^{4} \\
& +\frac{1}{2} \lambda u(t)^{2}+\frac{1}{4} \varepsilon_{3} u(t)^{4}+\frac{1}{6} \varepsilon_{4} u(t)^{6}
\end{aligned}
$$

Its Hamiltonian can be easily obtained, which reads:

$$
\begin{aligned}
H= & \frac{1}{2}\left(\frac{\mathrm{d}}{\mathrm{d} t} u(t)\right)^{2}+\frac{1}{2}\left(\frac{\mathrm{d}}{\mathrm{d} t} u(t)\right)^{2} \varepsilon_{1} u(t)^{2}+\frac{1}{2}\left(\frac{\mathrm{d}}{\mathrm{d} t} u(t)\right)^{2} \varepsilon_{2} u(t)^{4} \\
& +\frac{1}{2} \lambda u(t)^{2}+\frac{1}{4} \varepsilon_{3} u(t)^{4}+\frac{1}{6} \varepsilon_{4} u(t)^{6} .
\end{aligned}
$$

Integrating Eq. (31) with respect to $t$ from 0 to $T / 4$, we have:

$\bar{H}(u(t))=\int_{0}^{T / 4}\left\{\begin{array}{l}\frac{1}{2}\left(\frac{\mathrm{d}}{\mathrm{d} t} u(t)\right)^{2}+\frac{1}{2}\left(\frac{\mathrm{d}}{\mathrm{d} t} u(t)\right)^{2} \varepsilon_{1} u(t)^{2}+\frac{1}{2}\left(\frac{\mathrm{d}}{\mathrm{d} t} u(t)\right)^{2} \varepsilon_{2} u(t)^{4} \\ +\frac{1}{2} \lambda u(t)^{2}+\frac{1}{4} \varepsilon_{3} u(t)^{4}+\frac{1}{6} \varepsilon_{4} u(t)^{6}\end{array}\right\} \mathrm{d} t$

Assuming that the solution can be expressed as $u(t)=A \cos \omega t$ and substituting it to Eq. (32), we obtain:

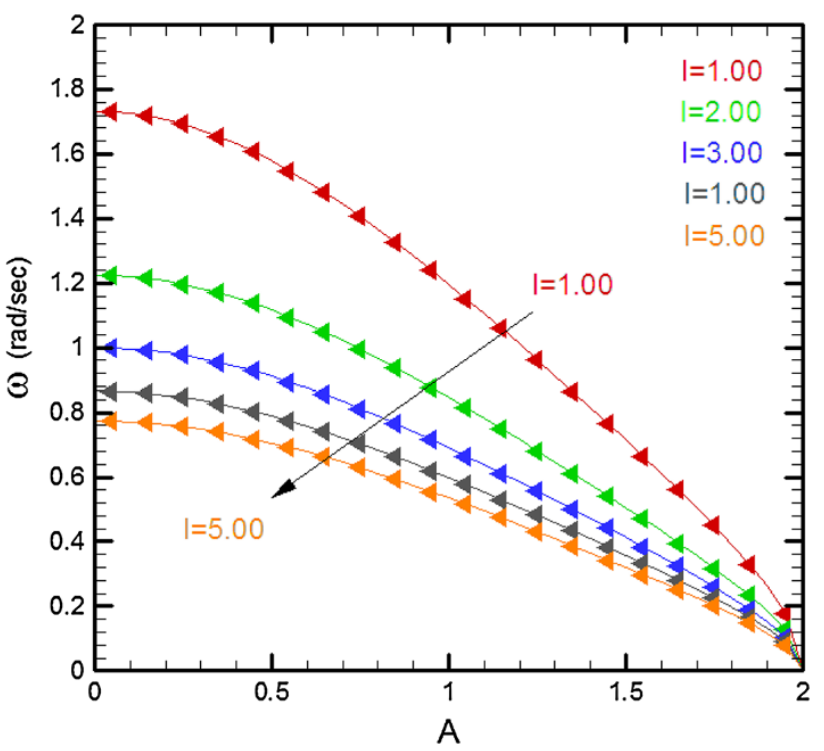

Fig. 4 Comparison of frequency corresponding to different values of amplitude $(A)$ and $g=1.00$

Setting:

$$
\begin{aligned}
\frac{\partial}{\partial A}\left(\frac{\partial \bar{H}}{\partial(1 / \omega)}\right)= & -A \omega^{2} \cdot \frac{\pi}{4}-2 \varepsilon_{1} A^{3} \omega^{2} \cdot \frac{\pi}{16}-3 \varepsilon_{2} A^{5} \omega^{2} \cdot \frac{\pi}{32} \\
& +\lambda A \cdot \frac{\pi}{4}+\varepsilon_{3} A^{3} \cdot \frac{3 \pi}{16}+\varepsilon_{4} A^{5} \cdot \frac{5 \pi}{32}=0 .
\end{aligned}
$$

We obtain the following frequency-amplitude relationship:

$\omega_{\mathrm{HAM}}=\sqrt{\frac{6 \varepsilon_{3} A^{2}+5 \varepsilon_{4} A^{4}+8 \lambda}{8+4 \varepsilon_{1} A^{2}+3 \varepsilon_{2} A^{4}}}$

$$
\begin{aligned}
& \bar{H}(u(t))=\int_{0}^{T / 4}\left\{\begin{array}{l}
\frac{1}{2} A^{2} \omega^{2} \sin ^{2} \omega t+\frac{1}{2} \varepsilon_{1} A^{4} \omega^{2} \sin ^{2} \omega t \cos ^{2} \omega t+\frac{1}{2} \varepsilon_{2} A^{6} \omega^{2} \sin ^{2} \omega t \cos ^{4} \omega t \\
+\frac{1}{2} \lambda A^{2} \cos ^{2} \omega t+\frac{1}{4} \varepsilon_{3} A^{4} \cos ^{4} \omega t+\frac{1}{6} \varepsilon_{4} A^{6} \cos ^{6} \omega t
\end{array}\right\} \mathrm{d} t \\
& =\int_{0}^{\pi / 2}\left\{\frac{1}{2} A^{2} \omega \sin ^{2} t+\frac{1}{\omega}\left[\begin{array}{l}
\frac{1}{2} \varepsilon_{1} A^{4} \omega^{2} \sin ^{2} t \cos ^{2} t+\frac{1}{2} \varepsilon_{2} A^{6} \omega^{2} \sin ^{2} t \cos ^{4} t \\
+\frac{1}{2} \lambda A^{2} \cos ^{2} t+\frac{1}{4} \varepsilon_{3} A^{4} \cos ^{4} t+\frac{1}{6} \varepsilon_{4} A^{6} \cos ^{6} t
\end{array}\right]\right\} \mathrm{d} t \\
& =\frac{1}{2} A^{2} \omega \cdot \frac{\pi}{4}+\frac{1}{\omega}\left[\begin{array}{l}
\frac{1}{2} \varepsilon_{1} A^{4} \omega^{2} \cdot \frac{\pi}{16}+\frac{1}{2} \varepsilon_{2} A^{6} \omega^{2} \cdot \frac{\pi}{32}+\frac{1}{2} \lambda A^{2} \cdot \frac{\pi}{4} \\
+\frac{1}{4} \varepsilon_{3} A^{4} \cdot \frac{3 \pi}{16}+\frac{1}{6} \varepsilon_{4} A^{6} \cdot \frac{5 \pi}{32}
\end{array}\right] .
\end{aligned}
$$


Table 2 Values of dimensionless parameters $\varepsilon_{i}$ for a mode

\begin{tabular}{lllll}
\hline Mode & $\varepsilon_{1}$ & $\varepsilon_{2}$ & $\varepsilon_{3}$ & $\varepsilon_{4}$ \\
\hline Mode-1 & 0.326845 & 0.232598 & 0.129579 & 0.087584 \\
Mode-2 & 1.642033 & 0.913055 & 0.313561 & 0.204297 \\
Mode-3 & 4.051486 & 1.665232 & 0.281418 & 0.149677
\end{tabular}

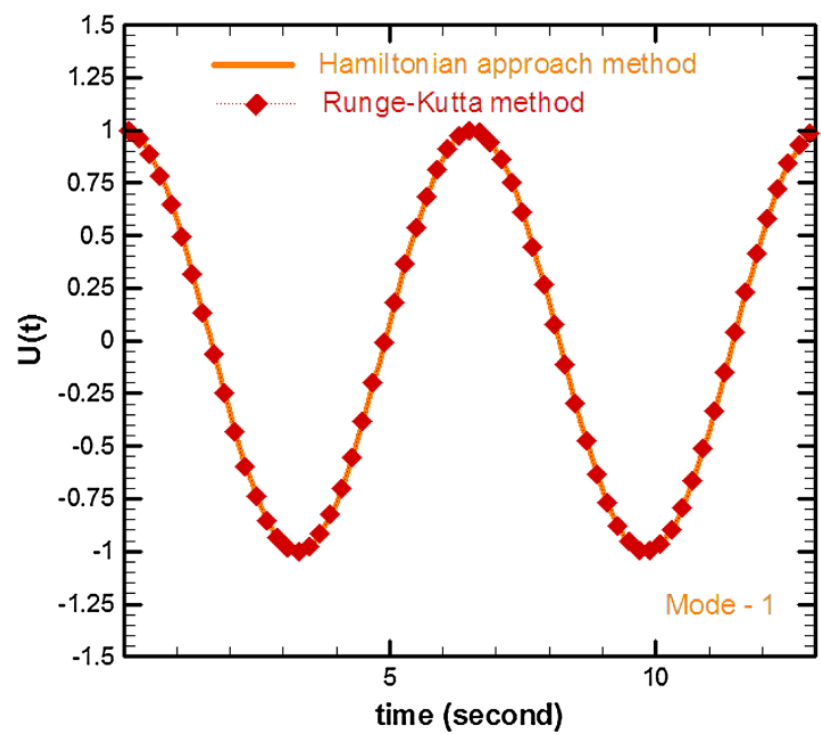

Fig. 5 Comparison of Hamiltonian approach method solution of $u(t)$ based on $t$ with numerical solution (Runge-Kutta method) for $\lambda=1.00, A=1.00$ in mode- 1

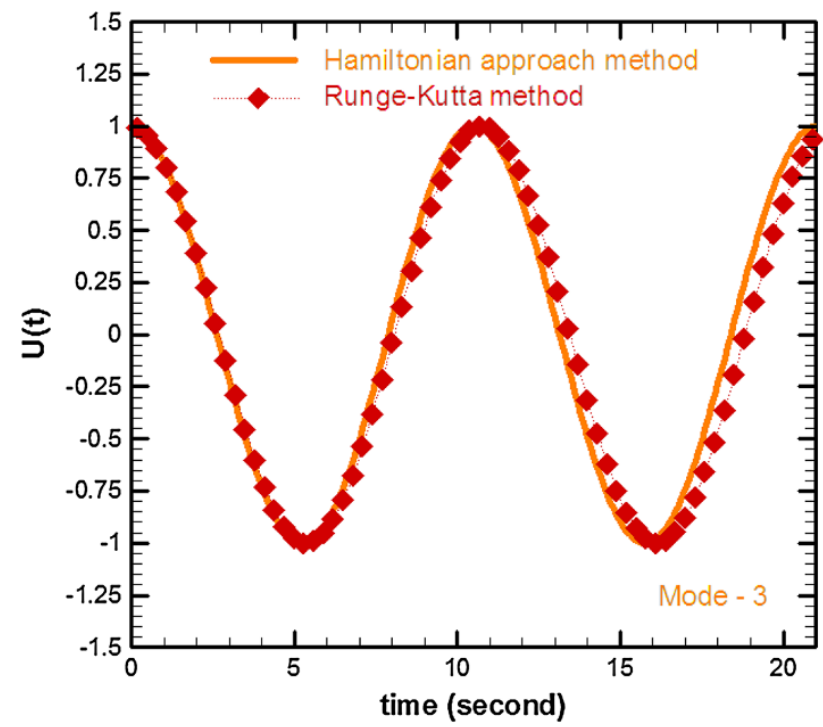

Fig. 6 Comparison of Hamiltonian approach method solution of $u(t)$ based on $t$ with numerical solution (Runge-Kutta method) for $\lambda=1.00, A=1.00$ in mode- 3

Its period can be written in the following form:

$$
T_{\mathrm{HAM}}=\frac{2 \pi}{\sqrt{\frac{6 \varepsilon_{3} A^{2}+5 \varepsilon_{4} A^{4}+8 \lambda}{8+4 \varepsilon_{1} A^{2}+3 \varepsilon_{2} A^{4}}}}
$$

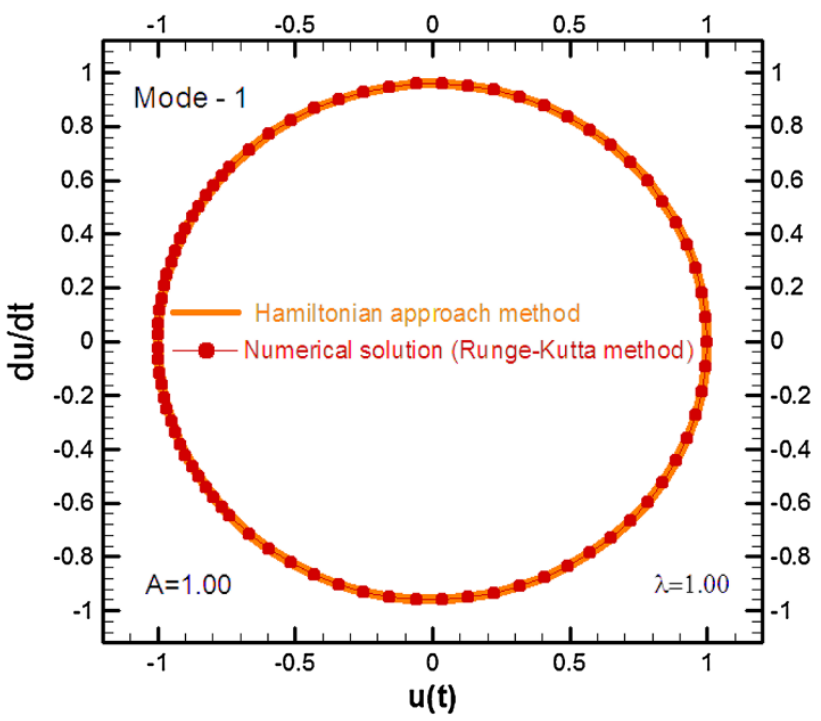

Fig. 7 Comparison of analytical approximate solution of $\mathrm{d} u / \mathrm{d} t$ based on $u(t)$ with the with numerical solution (Runge-Kutta method) for $\lambda=1.00, A=1.00$ in mode- 1

For this dynamical system, because there is no exact solution, we compare the approximate solution results with Runge-Kutta method, as one of the known numerical methods. The numerical solution with Runge-Kutta method for this nonlinear differential equation is:

$\frac{\mathrm{d}}{\mathrm{d} t} u_{1}(t)=u_{2}(t), \mathrm{u}_{1}(0)=A$

and

$$
\begin{aligned}
\frac{\mathrm{d}}{\mathrm{d} t} u_{2}(t)= & -\frac{1}{1+\varepsilon_{1} u_{1}(t)^{2}+\varepsilon_{2} u_{1}(t)^{4}} \\
& \left(\begin{array}{l}
\lambda u_{1}(t)+\varepsilon_{1} u_{1}(t) u_{2}(t)^{2} \\
+2 \varepsilon_{2} u_{1}(t)^{3} u_{2}(t)^{2} \\
+\varepsilon_{3} u_{1}(t)^{3}+\varepsilon_{4} u_{1}(t)^{5}
\end{array}\right) .
\end{aligned}
$$

Motion is assumed to start from the position of maximum displacement with zero initial velocity. $\lambda$ is an integer which may take values of $-1,0$ and 1 , and $\varepsilon_{1}, \varepsilon_{2}, \varepsilon_{3}$ and $\varepsilon_{4}$ are positive parameters. The values of parameters $\varepsilon_{1}, \varepsilon_{2}, \varepsilon_{3}$ and $\varepsilon_{4}$ associated for a mode are shown in Table 2.

To illustrate the accuracy of the Hamiltonian approach method solution, we present the comparison results of analytical approximate solution of $u(t)$ based on $t$ with the numerical solution which solved by Runge-Kutta method as one of the known numerical methods in Figs. 5 and 6 for $\lambda=1.00, A=1.00$ and various modes (mode-1 and mode-3).

Figures 7 and 8 present the high accuracy of Hamiltonian approach method solution in comparison with numerical solution for different values of $\varepsilon_{1}, \varepsilon_{2}, \varepsilon_{3}$ and $\varepsilon_{4}$, 


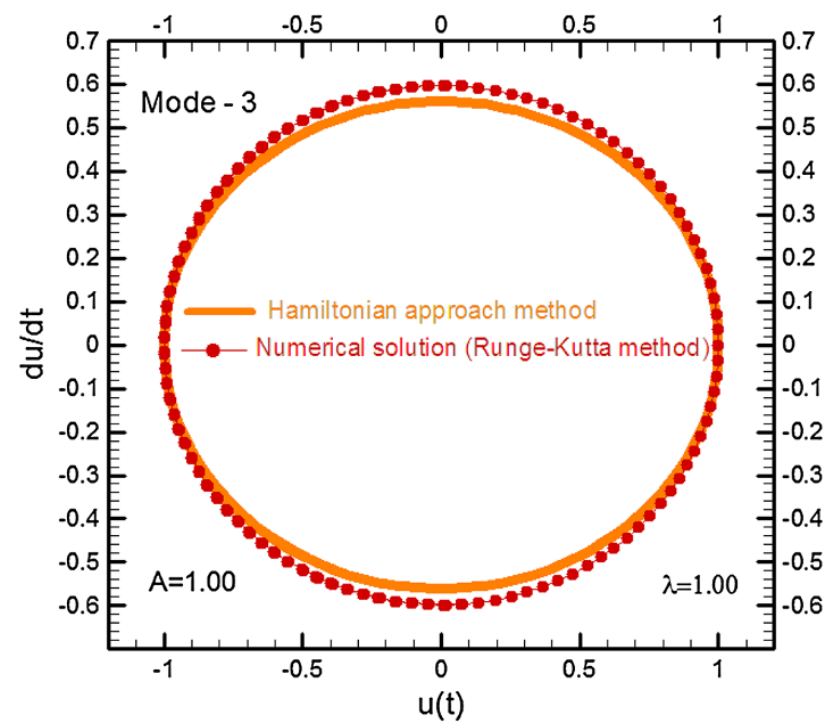

Fig. 8 Comparison of analytical approximate solution of $\mathrm{d} u / d t$ based on $u(t)$ with the with numerical solution (Runge-Kutta method) for $\lambda=1.00, A=1.00$ in mode- 3

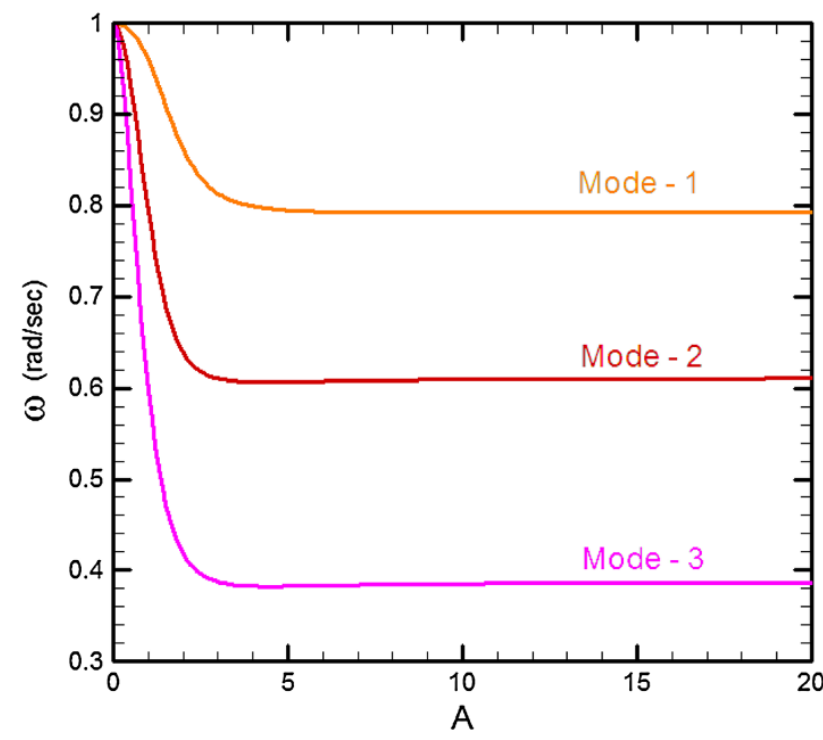

Fig. 9 Comparison of frequency corresponding to different values of amplitude $(A)$ and various modes, mode- 1 , mode- 2 and mode- 3

and show the phase-space curves ( $\mathrm{d} u / \mathrm{d} t$ versus $u(t)$ curve) for amplitude $A=1.00$ and $\lambda=1.00$.

It can be observed that the phase-space curves generated from approximate solution are close to that of the numerical curves. The phase plots show the behavior of the dynamical system in modes 1 and 3 . It is periodic with a center at $(0,0)$.

Also, to investigate on the behavior of this dynamical system, the effect of parameters $\varepsilon_{1}, \varepsilon_{2}, \varepsilon_{3}$ and $\varepsilon_{4}$ on the frequency corresponding to different values of amplitude (A) has been studied in Fig. 9.

It is evident that Hamiltonian approach method shows excellent agreement with the exact and numerical solutions and is quickly convergent and valid for a wide range of vibration amplitudes and initial conditions. The accuracy of the results shows that the Hamiltonian approach method can be potentiality used for the analysis of strongly nonlinear oscillation problems accurately.

\section{Conclusion}

In this article, we applied Hamiltonian approach method as one of the analytical approximate techniques, for studying the strongly nonlinear dynamical systems such as the motion of a rigid rod rocking back on the circular surface without slipping and the free vibrations of an autonomous conservative oscillator with inertia and static-type fifthorder nonlinearities.

Comparison of the results which are obtained by this method with the obtained result by the exact and numerical solutions reveal that the Hamiltonian approach method is very effective and convenient and does not require linearization or small perturbation and can be easily extended to other nonlinear dynamical systems and can therefore be found widely applicable in engineering and other sciences.

Authors contributions MH conceived the study and participated in its design and coordination. MS carried out the numerical solution of equations and participated in drafting the manuscript. HEK carried out the software work and the solution of equations using analytical approximate methods. All authors read and approved the final manuscript.

Acknowledgments This work was financially supported by the research deputy of Fakultät für Mathematik und Informatik, Friedrich-Schiller-Universität Jena, Germany. Authors would like to acknowledge them because of their support.

Conflict of interest The authors declare that they have no competing interests.

Open Access This article is distributed under the terms of the Creative Commons Attribution License which permits any use, distribution, and reproduction in any medium, provided the original author(s) and the source are credited.

\section{References}

1. Shou, D.H., He, J.H.: Application of parameter-expanding method to strongly nonlinear oscillators. Int. J. Nonlinear Sci. Numer. Simul. 8(1), 121-124 (2007)

2. Xu, L.: Determination of limit cycle by He's parameterexpanding method for strongly nonlinear oscillators. J. Sound Vib. 302(1), 178-184 (2007)

3. Xu, L.: Application of He's parameter-expanding method to an oscillation of a mass attached to a stretched elastic wire. Phys. Lett. A 368(3), 259-262 (2007) 
4. He, J.H., XH, W.: Variational iteration method: new development and applications. Comput. Math. Appl. 54(7), 881-894 (2007)

5. Shou, D.H., He, J.H.: Beyond Adomian method: the variational iteration method for solving heat-like and wave-like equations with variable coefficients. Phys. Lett. A 372(3), 233-237 (2008)

6. Shou, D.H., He, J.H.: Variational iteration method for two-strand yarn spinning. Nonlinear Anal. Theory, Methods Appl. 71(12), 830-833 (2009)

7. He, J.H.: A short remark on fractional variational iteration method. Phys. Lett. A 375(38), 3362-3364 (2011)

8. He, J.H.: Preliminary report on the energy balance for nonlinear oscillations. Mech. Res. Commun. 29(2), 107-111 (2002)

9. Ganji, D.D., Afrouzi, G.A., Talarposhti, R.A.: He's energy balance method for nonlinear oscillators with discontinuities. Int. J. Nonlinear Sci. Numer. Simul. 10(3), 301-304 (2009)

10. Zhang, H.L., Xu, Y.G., Chang, J.R.: Application of He's energy balance method to a nonlinear oscillator with discontinuity. Int. J. Nonlinear Sci. Numer. Simul. 10(2), 207-214 (2009)

11. Ganji, D.D., Ganji, S.S., Karimpour, S.: He's energy balance and He's variational methods for nonlinear oscillations in engineering. Int. J. Mod. Phys. B 23(3), 461-471 (2009)

12. Ebrahimi Khah, H., Ganji, D.D.: A study on the motion of a rigid rod rocking back and cubic-quintic duffing oscillators by using He's energy balance method. Int. J. Nonlinear Sci. 10(4), 447-451 (2010)

13. Ebrahimi Khah, H., Ayazi, A., Lale Arefi, S.H.: Approximate analytical solution to study of nonlinear oscillator with discontinuity using energy balance method. In: Proceedings of 42th Annual Iranian Mathematics, Conference, 5-8 Sept 2011, Vali-eAsr University of Rafsanjan, Iran

14. Ebrahimi Khah, H., Ayazi, A., Ganji, D.D.: A study on the forced vibration of strongly nonlinear oscillator using He's energy balance method. J. Adv. Res. Mech. Eng. 2(1), 27-32 (2011)

15. He, J.H.: Variational approach for nonlinear oscillators. Chaos Solitons Fractals 34(5), 1430-1439 (2007)

16. Shou, D.H.: Variational approach to the nonlinear oscillator of a mass attached to a stretched wire. Phys. Scr. 77(4), 045006 (2008)

17. Shou, D.H.: Variational approach for nonlinear oscillators with discontinuities. Comput. Math. Appl. 58(11), 2416-2419 (2009)

18. Ebrahimi Khah, H., Ganji, D.D.: Application of He's variational approach method for strongly nonlinear oscillators. J. Adv. Res. Mech. Eng. 1(1), 30-34 (2010)

19. Ebrahimi Khah, H., Ayazi, A., Daie, M.: A study on the free oscillation of pendulum using variational approach method and comparison with exact solution. In: Proceedings of 41th Iranian International Conference on Mathematics, 12-15 Sept 2010, University of Urmia, Urmia, Iran

20. He, J.H.: An improved amplitude-frequency formulation for nonlinear oscillators. Int. J. Nonlinear Sci. Numer. Simul. 9(2), 211-212 (2008)

21. He, J.H.: Asymptotic methods for solitary solutions and compactions. In: Proceedings of Abstract and Applied Analysis. Hindawi Publishing Corporation, Hindawi (2012). doi:10.1155/ 2012/916793

22. Ebrahimi Khah, H., Ayazi, A., Ganji, D.D.: Determination of the amplitude frequency for strongly nonlinear oscillator with using two approximate analytical techniques. J Theor. Appl. Phys. (2013). doi:10.1186/2251-7235-7-38

23. He, J.H.: Homotopy perturbation technique. Comput. Methods Appl. Mech. Eng. 178, 257-262 (1999)

24. He, J.H.: Recent development of the homotopy perturbation method. Topol. Methods Nonlinear Anal. 31(2), 205-209 (2008)

25. He, J.H.: An elementary introduction to the homotopy perturbation method. Comput. Math. Appl. 57(3), 410-412 (2009)

26. Shou, D.H.: The homotopy perturbation method for nonlinear oscillators. Comput. Math. Appl. 58(11), 2456-2459 (2009)
27. Ebrahimi Khah, H., Ayazi, A., Ganji, D.D.: The investigation and application of two approximate analytical methods for the solution of nonlinear differential equation of beam elastic deformation. J. Theor. Appl. Phys. 5(2), 53-58 (2011)

28. Ebrahimi Khah, H., Hermann, M., Saravi, M.: The comparison of homotopy perturbation method with finite difference method for determination of maximum beam deflection. J. Theor. Appl. Phys. (2013). doi:10.1186/2251-7235-7-8

29. Ebrahimi Khah, H., Ayazi, A., Lale Arefi, S.H.: The solution of beam deformation equation using homotopy perturbation method.In: Proceedings of 42th Annual Iranian Mathematics Conference, 5-8 Sept 2011, Vali-e-Asr University of Rafsanjan, Iran

30. He, J.H.: Some asymptotic methods for strongly nonlinear equations. Int. J. Mod. Phys. B 20(10), 1141-1199 (2006)

31. He, J.H.: Hamiltonian approach to nonlinear oscillators. Phys. Lett. A 374(23), 2312-2314 (2010)

32. He, J.H., Zhong, T., Tang, L.: Hamiltonian approach to duffingharmonic equation. Int. J. Nonlinear Sci. Numer. Simul. 11(1), 43-46 (2010)

33. Xu, L., He, J.H.: Determination of limit cycle by Hamiltonian approach for strongly nonlinear oscillators. Int. J. Nonlinear Sci. Numer. Simul. 11(12), 1097-1101 (2010)

34. He, J.H., Kong, H.Y., Yang, R.R., et al.: Review on fiber morphology obtained by bubble electro spinning and blown bubble spinning. Thermal Sci. 16(5), 1263-1279 (2012)

35. He, J.H.: Asymptotic methods for solitary solutions and compactions. In: Proceedings of Abstract and Applied Analysis. Hindawi Publishing Corporation, Hindawi (2012). doi:10.1155/ 2012/916793

36. Wu, B.S., Lim, C.W., He, J.H.: A new method for approximate analytical solutions to nonlinear oscillations of non-natural systems. Nonlinear Dyn. 32(1), 1-13 (2003)

37. Hamden, M.N., Shabaneh, N.H.: Study on the large amplitude free vibrations of a restrained uniform beam carrying an intermediate lumped mass. J. Sound Vib. 199(6), 711-736 (1997)

M. Hermann was born in 1949 , in the city of Weimar, Germany. He is a reader of numerical analysis in the Friedrich-Schiller-Universität Jena in Germany and published several papers and books on numerical solutions of differential equations. At present, he is supervisor of several M.Sc. and Ph.D. students at the University of Schiller.

M. Saravi was born in 1953, in the city of Amol, Iran. He started off academic studies in UK's Dudley Technical College. He received his first degree in Mathematics and Statistics from Polytechnic of North London, and his second degree in Numerical Analysis from Brunel University and his Ph.D. at UK's Open University. His research interests include numerical solution of ODEs, differential equations, and integral equations as well as DAE and spectral methods. He is currently a board member at IAU, Iran.

H. Ebrahimi Khah was born in Tehran, Iran on September 7, 1984. He received his B.Sc. degree in Civil Engineering-Building from the Shomal University, Amol, Iran in 2006 and M.Sc. degree in Civil Engineering-Structure from the Shomal University in Amol, Iran in 2008. At present, he is doing his researches toward Ph.D. degree in Friedrich Schiller University of Jena, Germany. He has published several technical manuscripts in refereed scientific journals. His research has concentrated on numerical and analytical approximate solutions of strongly nonlinear ODEs and PDEs, dynamical systems, and structural deformations. 